\title{
Impedance Spectroscopy of Bilayer Lipid Membranes Self- assembled on Agar Support - Interaction with HDL
}

\author{
M. LEGIŇ, G. LAPUTKOVÁ, J. SABO, L. VOJČÍKOVÁ \\ Department of Medical Biophysics, Faculty of Medicine, Pavol Jozef Šafárik University, Košice, \\ Slovakia
}

Received May 23, 2007

Accepted May 29, 2007

On-line available May 31, 2007

\begin{abstract}
Summary
The electrical properties of the supported lipid bilayer membrane (s-BLM) of egg phosphatidylcholine (PC) selfassembled on agar surface were examined. To characterize the insulating properties of s-BLMs, electrochemical impedance spectroscopy (EIS) was used. The analysis of impedance spectra in terms of an equivalent circuit of agar/electrolyte and agar/s-BLM/electrolyte in the frequency range of $0.1 \mathrm{~Hz}-10 \mathrm{kHz}$ was performed. The high-density lipoproteins (HDL)/s-BLM interaction in the concentration range from $20 \mu \mathrm{g} / \mathrm{ml}$ to $80 \mu \mathrm{g} / \mathrm{ml}$ of HDL was investigated. It is evident that treatment of s-BLM with HDL resulted in an increase of the lipid film resistance and a decrease of membrane capacitance.
\end{abstract}

\section{Key words}

Agar-supported bilayer membrane $\bullet$ High-density lipoprotein $\bullet$ Electrochemical impedance spectroscopy

\section{Introduction}

An electrode-agar gel system as a support for the fabrication of s-BLM was used here. Agar surfaces offer a wide range of attractive physical properties that are important for the design of technical applications (Matsuno et al. 2004). Agar support allows overcoming one of the main drawbacks of the membrane systems on solid substrates - asymmetry of the two layers of the membrane. Other properties, such as fluidity and selfsealing, make them similar to the conventional BLMs.

Agar s-BLM can be accessed by a variety of surface analysis tools. In a number of reports it has been shown that EIS is useful as a nondestructive and sensitive method for investigation of lipid films on solid surfaces
(Wiegand 2000). The theoretical analysis of EIS data by fitting to a model represented by an equivalent electrical circuit allows the understanding of processes associated with the biomaterial-functionalized electrode surfaces.

The motivation of the present work was the implementation of EIS as an investigative tool for agar sBLM/HDL interaction. HDL are highly heterogeneous lipoprotein particles consisting of an approximately equal amount of protein and lipid, mainly phospholipids and unesterified cholesterol. As a result of qualitative and quantitative differences in lipid, protein and enzyme content, HDL particles are multi-shaped complexes with varying density, fluidity, and charge. They consist of a hydrophobic core surrounded by a surface monolayer of phospholipids, unesterified cholesterol and apolipo- 
proteins. HDL apolipoproteins have amphipathic properties, conferred by regions containing both polar and nonpolar amino acid residues. Hydrophobic forces whereby the fatty acyl chains and the nonpolar amino acids side chains are excluded from the aqueous environment drive the association of polar lipids and apolipoproteins in HDL. The apolipoproteins and the unesterified cholesterol have appreciable water solubility and can exchange easily between lipoproteins or with other lipid surfaces (Calabresi et al. 2006). The height of the HDL complex is consistent with a phospholipid bilayer structure. Furthermore, it has been demonstrated (Carlson et al. 1997) that HDL fuse when adsorbed to mica. Even if HDL binding proteins identified in the plasma membrane (Graham and Oram 1987) have been considered essential for HDL-membrane interaction, some studies indicate that HDL can interact with a protein-free lipid bilayer (Johnson et al 1988, Tricerri et al. 1998). It is important to emphasize that most of HDL functions are exerted not by most apoproteins, but rather by lipids or enzymes associated with HDL (Nofer et al. 2002).

\section{Methods}

\section{Materials}

L- $\alpha$-Phosphatidylcholine from egg yolk and ndodecane were purchased from Sigma. The membrane forming solution was prepared by mixing an ethanol solution of PC with n-dodecane to attain a final $5 \%$ lipid concentration. Lyophilised HDL from human plasma (Fluka) were rehydrated by lipoprotein refolding buffer (Fluka). Other chemicals ( $\mathrm{NaCl}$, Tris, EDTA) were of p.a. quality, and were used without further purification. The water used was Millipore-filtered $(\mathrm{R}>18 \mathrm{M} \Omega . \mathrm{cm}$, $\mathrm{pH}$ 5.5). As a standard buffer for electrochemical measurements, an aqueous solution of $150 \mathrm{mmol} / \mathrm{l} \mathrm{NaCl}$ containing $10 \mathrm{mmol} / 1$ Tris and $1 \mathrm{mmol} / \mathrm{l}$ EDTA was used.

\section{Method for supported lipid layer formation}

The formation of s-BLM was in accordance with the procedure developed and further optimized by Lu et al. (1996). An agar-filled Teflon tube was used as a solid substrate. The tube was cut with a scalpel and dipped in a membrane-forming solution for about $1 \mathrm{~s}$. Subsequently the tip was immersed in an aqueous solution. The exposure to the electrolyte promoted an alignment of the amphiphilic molecules leading to the formation of a bilayer.

\section{Electrochemical measurements}

$\mathrm{AC}$ analysis was carried out with a Zahner IM6e (Kronach) electrochemical analyzer providing fully computer-controlled EIS. The absolute values of the impedance $|Z(f)|$ and the phase shift angle $\varphi(f)$ were recorded in a frequency range from $10^{-1}$ to $10^{4} \mathrm{~Hz}$. A small sinusoidal voltage of $20 \mathrm{mV}$ was applied and the current response of the system was measured. EIS data were analyzed by means of complex non-linear regression least-square (CNRLS) fit to a model represented by an equivalent electrical circuit. The data analysis was done using THALES software from Zahner Elektrik. A three-electrode system was used for the EIS experiments. A Teflon tube with the inner diameter of $0.5 \mathrm{~mm}$ filled with $3 \%$ agar in $0.15 \mathrm{M} \mathrm{KCl}$ with an $\mathrm{Ag} / \mathrm{AgCl}$ wire was used as the working electrode, a platinum wire served as a counter electrode, and $(\mathrm{Ag} / \mathrm{AgCl}, 3.5 \mathrm{M} \mathrm{KCl})$ as the reference electrode. The thermostatically controlled measuring cuvette placed into a Faraday cage was used. All experiments were carried out at room temperature $(21.0 \pm 0.2){ }^{\circ} \mathrm{C}$.

\section{Results}

Due to the suitable properties of agar surfaces to serve as a matrix for the formation of symmetrical lipid membranes, the EIS response of the bare agar-filled working electrode was studied first. Fig. 1 gives the Nyquist plot of the impedance spectrum with the inset of an equivalent electrical circuit for fitting the measured data. In the studied frequency range the spectrum is composed of an elongated semicircle at high frequencies prolonged by a curved line for low frequencies. The semicircle corresponds to the contribution of the resistance of the agar phase $(\mathrm{R})$ in parallel with its capacitance represented with the constant phase element CPE due to the non-ideal capacitive response of the interface. CPE generates the impedance that can be expressed as follows:

$Z_{\mathrm{CPE}}(j \omega)=1 / Y_{0}(j \omega)^{\alpha}$

where $0<\alpha<1$ is the CPE power and $Y_{0}$ is the CPE constant. $\omega$ is the angular frequency; $j$ is the imaginary unit (McDonald 1987). The curved line should be ascribed to the $\mathrm{CPE}_{\mathrm{d}}$ capacitance of the double-layer of the electrolyte/agar interface. This behavior is, basically, explained by diffusion charge transport that is dominant at lower frequencies. $\mathrm{CPE}_{\mathrm{d}}$ behavior is intensified by 
Table 1. Fitted data for the agar support after modification with $P C$ and the addition of HDL

\begin{tabular}{llllll}
\hline $\mathbf{H D L}(\boldsymbol{\mu g} / \mathbf{m l})$ & $\boldsymbol{C}_{\mathbf{d}}(\mathbf{p F})$ & $\boldsymbol{R}(\mathbf{M} \boldsymbol{\Omega})$ & $\boldsymbol{C}(\mathbf{p F})$ & $\boldsymbol{R}_{\mathbf{e}}(\mathbf{k} \boldsymbol{\Omega})$ & $\boldsymbol{C}_{\mathbf{s}}(\mathbf{p F})$ \\
\hline 0 & $320.3 \pm 57.9$ & $82.2 \pm 23.7$ & $50.7 \pm 4.5$ & $11.1 \pm 0.1$ & $45.0 \pm 0.3$ \\
20 & $345.8 \pm 75.9$ & $136.9 \pm 49.0$ & $46.1 \pm 4.6$ & $11.1 \pm 0.2$ & $45.5 \pm 0.3$ \\
40 & $430.3 \pm 104.6$ & $157.3 \pm 57.4$ & $42.1 \pm 4.1$ & $11.2 \pm 0.1$ & $45.6 \pm 0.4$ \\
60 & $436.7 \pm 108.4$ & $199.0 \pm 60.9$ & $38.7 \pm 2.1$ & $11.1 \pm 0.1$ & $45.4 \pm 0.5$ \\
80 & $504.9 \pm 74.8$ & $242.1 \pm 48.7$ & $38.6 \pm 2.6$ & $11.4 \pm 0.3$ & $45.3 \pm 0.2$ \\
\hline
\end{tabular}

inhomogeneities in the dimension and the shape of the capillary pores in agar. Resistance $R_{\mathrm{e}}$ in serial combination represents the contribution from the bulk electrolyte, contacts, and connections. The equivalent circuit was extended to take into account the capacitance $C_{\mathrm{s}}$ representing the stray capacitance of the measuring cell. Ideally, $R_{\mathrm{e}}$ and $C_{\mathrm{s}}$ are not affected by modification on the electrode surface as they represent the properties of the electrolyte solution and the measuring cell.

The quality of fitting was evaluated by the error value. By using the CPE, the quality of fitting had a decreased error value; the mean relative error of $|Z|$ did not exceed $0.1 \%$, the maximum error of $\varphi$ did not overstep $0.3 \mathrm{deg}$. The fitting procedure yielded for the bare electrode a CPE capacitance value of $C=53.4 \mathrm{pF}$ and a resistance value of $R=85.4 \mathrm{k} \Omega$. The value of a $\mathrm{CPE}_{\mathrm{d}}$ capacitance was determined as $C_{\mathrm{d}}=379.2 \mathrm{nF}$. The disturbing capacitance for the entire circuit reached a value of $C_{\mathrm{s}}=44.5 \mathrm{pF}$ and an electrolyte resistance corresponding to the value of $R_{\mathrm{e}}=11.7 \mathrm{k} \Omega$.

The self-assembly of s-BLM was followed by means of monitoring the change of the modulus of the electrical impedance $|Z|$ and the phase shift angle $\varphi$ of the system at $10 \mathrm{kHz}$ sinusoidal voltage. The time for the lipid film stabilization was of the order of $30 \mathrm{~min}$. This time is less than that obtained for Pt-supported membrane (Sabo et al. 1997), but it is twice as much as the stabilization of agar-thin film s-BLM described by Novotny et al. (1997). It should be mentioned that membrane formation strongly depended on the quality of the Teflon rim. If the rim was poorly cut, membrane formation was difficult and a rupture occurred within a few minutes. The lifetime of s-BLMs was rarely less than $24 \mathrm{~h}$.

The lipid film changed the impedance spectra quite markedly, showing the formation of a densely packed lipid membrane. In Fig. 2 a typical spectrum of sBLM is shown. Electrical parameters of s-BLM were obtained from fitting the parameters of the equivalent circuit shown in Fig. 1 to the data. The circuit used is formally the same as that used for the bare agar electrode It should be mentioned that now the CPE capacitance $C$ of the supported system covered with lipid contained contributions of both the lipid layer capacitance $C_{\mathrm{m}}$ and the capacitance of the agar phase $C_{\mathrm{a}}$ of the bare electrode (Ding et al. 2002). The two capacitances were in series and hence the CPE capacitance $C$ could be described by the following expression:

$1 / C=1 / C_{\mathrm{m}}+1 / C_{\mathrm{a}}$

Similarly, the resistance $R$ follows the expression:

$R=R_{\mathrm{m}}+R_{\mathrm{a}}$

As for the bare agar electrode $\mathrm{CPE}_{\mathrm{d}}$ behavior represents the charge transfer at the agar surface, now restricted by the deposition of the lipid layer.

The mean relative error of $|Z|$ did not exceed $0.5 \%$, the mean error of $\varphi$ did not overstep 0.2 deg for sBLM spectra. The fitting procedure yielded a CPE capacitance value of $C=50.7 \mathrm{pF}$ and a resistance value of $R=82.2 \mathrm{M} \Omega$. The obtained value of CPE power $\alpha$ (about ${ }^{\circ} 0.8$ ) demonstrates a deviation of the system from ideal capacitive response and confirms the suitability of CPE for modeling the electrolyte/electrode interfaces. The value of a $\mathrm{CPE}_{\mathrm{d}}$ capacitance was determined as $C_{\mathrm{d}}=$ $320.3 \mathrm{pF}$. As expected, the disturbing capacitance for the entire circuit and an electrolyte resistance did not differ significantly from the values obtained for the bare agar surface. Simulated values of the main electrical elements in the equivalent circuit are given in Table 1 as means \pm $\mathrm{SD}(n=6)$.

Following expression (2) the contribution from the membrane capacitance $C_{\mathrm{m}}$ calculated from the fitted values of the capacitance $C$ and the capacitance of the 


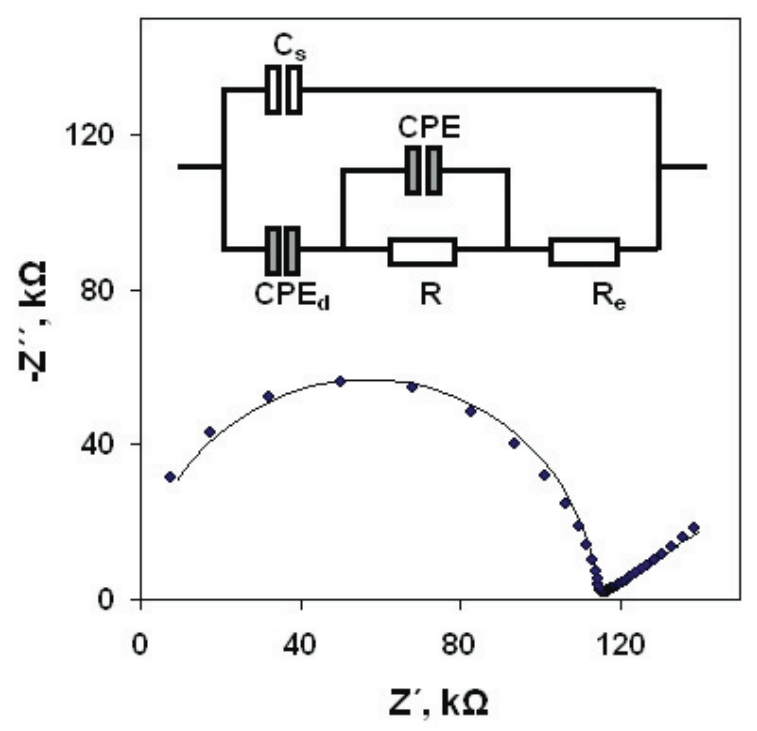

Fig. 1. The Nyquist plot of the impedance spectrum for the bare agar electrode. The solid line is the result of the fitting procedure using the equivalent circuit. Frequency range: $0.1 \mathrm{~Hz}-10 \mathrm{kHz}$. Amplitude of AC voltage: $20 \mathrm{mV}$. Inset: Equivalent circuit used to model impedance data.

agar phase of the bare agar electrode $C_{\mathrm{a}}$ comprises $\approx 880 \mathrm{pF}$, which represents the value corresponding to the well-developed lipid bilayer for the support area of $1.96 \times$ $10^{-3} \mathrm{~cm}^{2}$.

HDL were used to investigate the functionality of s-BLM immobilized on agar surface. It is known that low HDL $(<35 \mu \mathrm{g} / \mathrm{ml})$ may be associated with the increased risk of the coronary atherosclerosis, while conversely high levels $(>55 \mu \mathrm{g} / \mathrm{ml})$ appear to have a protective effect. EIS response of agar s-BLM was observed in the concentration range from 20 to $80 \mu \mathrm{g} / \mathrm{ml}$ of HDL. Fig. 2 shows impedance spectra at different HDL concentrations. The Nyquist plots provide a more direct and easy understanding of the conduction processes involved. It can be noticed that the areas enlarge with increasing HDL.

Analysis of the fitted data (Table 1) revealed that the major changes were those in the capacitance $C$ of the constant phase element $\mathrm{CPE}$ and the resistance $R$. The change of $\mathrm{CPE}_{\mathrm{d}}$ capacitance $C_{\mathrm{d}}$ was observed as well. Addition of HDL resulted in a decrease of the capacitance $C$ and an increase of the resistance $R$. Approximately 15 min after HDL addition the impedance spectra remained constant (not shown). The overall effect of HDL on the electrical parameters of s-BLM was more pronounced for the resistance $R$. When the concentration of HDL in the electrolyte reached $80 \mu \mathrm{g} / \mathrm{ml}$, the change in the resistance $R(195 \%)$ was much higher than that obtained for the

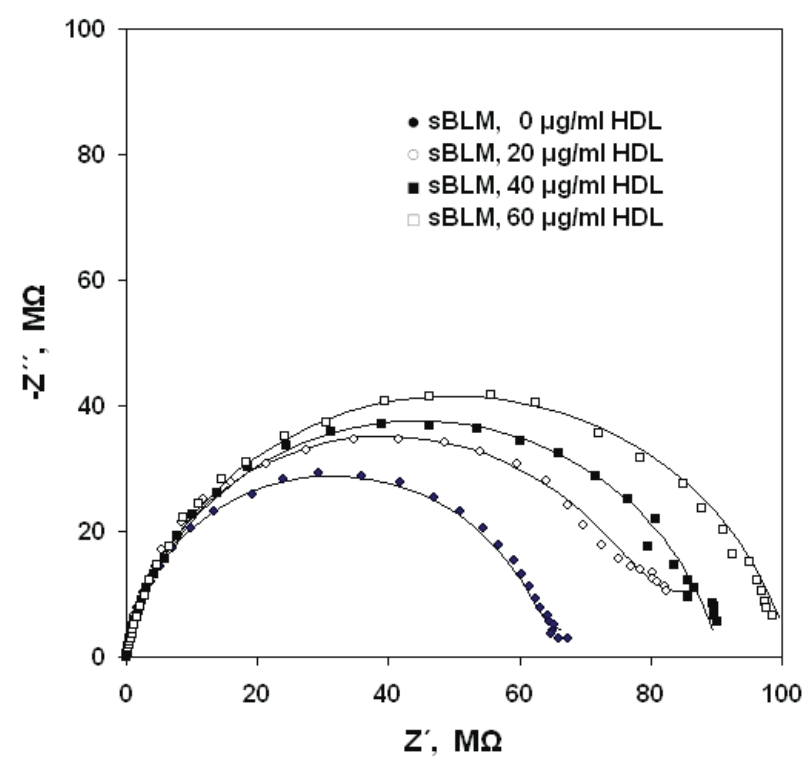

Fig. 2. Nyquist plots of the impedance spectra for the s-BLM on the agar support with the addition of HDL. The solid lines are the result of fitting the parameters of the equivalent circuit shown in Fig. 1. Frequency range: $0.1 \mathrm{~Hz}-10 \mathrm{kHz}$. Amplitude of $\mathrm{AC}$ voltage: $20 \mathrm{mV}$.

capacitance (33\%), indicating that $R$ could be more effective in the detection of the HDL/s-BLM interaction compared with the CPE capacitance $C$.

As the change of the CPE capacitance $C$ is indicative of the possible change of the thickness of the deposited membrane, the dependence in Fig. 3 shows the behavior of the s-BLM capacitance $C_{\mathrm{m}}$ over the studied HDL concentration range. A steep decrease of membrane capacitance observed for the low concentration was followed by a subtle change of the membrane capacitance over $60 \mu \mathrm{g} / \mathrm{ml}$.

For further illustration the meaning of the fitted impedance data, the calibration plots of the CPE capacitance $C_{\mathrm{m}}$ and the resistance $R$ were made. Fig. 4 exhibits the calibration plot of the difference in the inverse capacitance $C_{\mathrm{m}}$ after and before HDL interacted with the lipid film, $\Delta\left(1 / \mathrm{C}_{\mathrm{m}}\right)$, as a function of $\mathrm{HDL}$ concentration. While the plot shows for the lowest HDL concentrations a linear relationship, for higher concentrations the gradual saturation of the curve was observed. Fig. 5 shows the calibration plot of the difference in the resistance $\Delta R$ as a function of HDL concentration. Like for the capacitance, a deflection from linear dependence was observed for higher HDL concentrations. As revealed by the evaluation of the impedemetric data, the resistance signal is quite sensitive for the study of the HDL/s-BLM interaction as the calculated sensitivity reached the value of $1.5 \mathrm{M} \Omega / \mu \mathrm{g} / \mathrm{ml}$. 


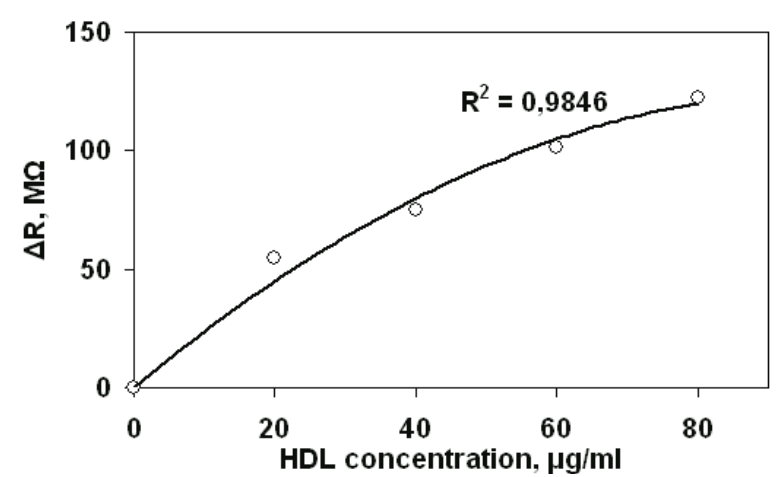

Fig. 3. Response of s-BLM capacitance $C_{m}$ to HDL concentrations.

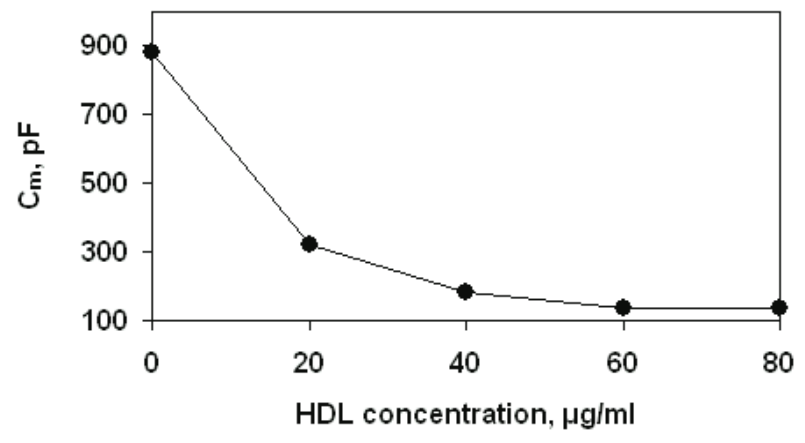

Fig. 4. Changes in the inverse $\mathrm{CPE}$ capacitance $\Delta\left(1 / C_{\mathrm{m}}\right)=$ $1 / C_{\mathrm{mHDL}}-1 / C_{\mathrm{m}}$ of s-BLM as a function of HDL concentration.

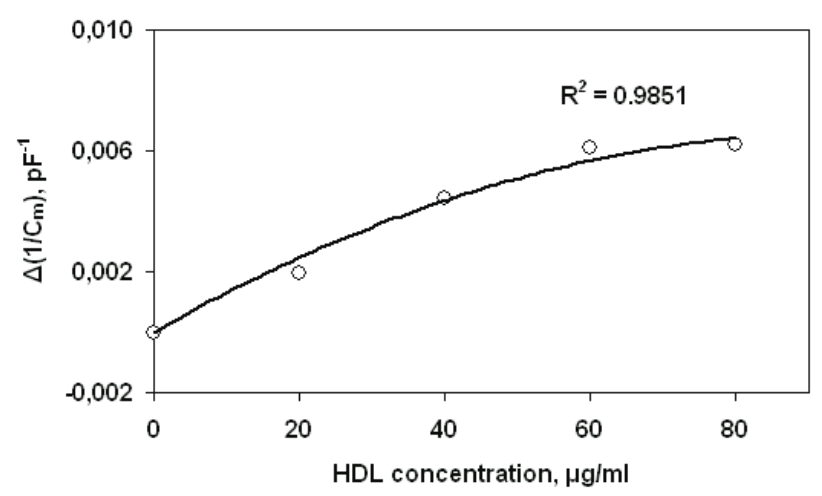

Fig. 5. Changes in the resistance $\Delta R=R_{\mathrm{HDL}}-R$ of the lipid bilayer on the agar as a function of HDL concentration.

\section{Discussion}

Agar (agarose) intensively used recently as a support makes it possible to prepare the stable thin membrane separating two aqueous media that are viscous, fluid and polar enough possessing the electrical properties of the conventional BLM. If the major drawback of free-standing BLMs is their low long-term stability, usually not exceeding $8 \mathrm{~h}$ (Tien and Ottova 1999), s-BLMs reported here exhibited significantly higher mechanical and long-term robustness, lasting rarely for less than $24 \mathrm{~h}$. The subsequent rupture of sBLM may be due to hydrolysis of the ester linkage of the glycerol residue of natural phospholipid in the presence of water (Costello et al. 1999). The products of the hydrolysis are soluble and diffuse into the agar gel.

The contribution from membrane capacitance $C_{\mathrm{m}}$ $(\approx 880 \mathrm{pF})$ calculated from the fitted values of the capacitance $C$ and the capacitance of the agar phase of the bare agar electrode $C_{\mathrm{a}}$ represents the value corresponding to the well-developed lipid bilayer for the support area of $1.96 \times 10^{-3} \mathrm{~cm}^{2}$. It is close to those values of the specific membrane capacitance of BLM. The highest value of the specific capacitance of BLM reported is $1.5 \mu \mathrm{F} / \mathrm{cm}^{2}$, although most researchers use an upper limit of $1.0 \mu \mathrm{F} / \mathrm{cm}^{2}$. The lower limit of $0.3 \mu \mathrm{F} / \mathrm{cm}^{2}$, and values in this range, corresponds to bilayers that are highly expanded, probably containing organic solvent between the phospholipid tails (White 1985).

The analysis of impedance data led to the arearelated membrane resistance of $\approx 0.16 \mathrm{M} \Omega . \mathrm{cm}^{2}$ that is comparable well with other types of s-BLMs reported (Bordi et al. 2002). However, it is lower than the specific membrane resistance of the free-standing planar lipid bilayers, which exhibit very high resistance of the order of $10^{7}-10^{8} \Omega . \mathrm{cm}^{2}$ (Mueller and Rudin 1968).

Surface roughness and porosity of the electrode surface have been considered as important factors contributing to CPE behavior. It seems possible that the low value of CPE power $\alpha$ results from the increased roughness of the lipid film due to the disturbing effect of chains of the agarose polymer.

According to Wang et al. (2002), the CPE behavior is also due to diffusion, which is increased by inhomogeneities in the dimension and shape of the capillary pores. $\mathrm{CPE}_{\mathrm{d}}$ values obtained for the bare agar electrode and s-BLM support the idea that the deposition of the membrane led to the substantial decrease of $\mathrm{CPE}_{\mathrm{d}}$ capacitance.

Finally, it is worth mentioning that the equivalent circuit selected for fitting does not meet all requirements, especially those regarding the direct extraction of the s-BLM capacitance $C_{\mathrm{m}}$ and the resistance $R_{\mathrm{m}}$ and needs additional improvement.

The second aim of this work was to study the interaction of human plasma HDL with s-BLM free of cholesterol. The interaction showed a response of the system proportional to the concentration of HDL. A decrease of the CPE capacitance $C$ and, at the same time, an increase of the resistance $R$ was observed. It is 
plausible that the increase of the membrane resistance or the decrease of a leakage current after addition of HDL could be conceivably caused by a thickening process. The possibility cannot be ruled out because the membrane system capacitance is observed simultaneously to decrease. The improved leakage properties at low HDL concentration can result from an annealing process, in which the holes in the bilayer are gradually filled in. A decrease in the capacitance at the higher HDL concentrations reaches a value that is indicative of an eventual adsorption of HDL on the bilayer creating multilayer structures.

The increased amount of water molecules displaced from the lipid layer due to the annealing process could give rise to the mean dielectric constant in the interspace. Then the change of $\mathrm{CPE}_{\mathrm{d}}$ capacitance, due to the added HDL, can result from the change in the mean dielectric constant in the space between agar and the lipid layer.

It has been proposed that cholesterol transport from plasma membranes to acceptor HDL could be modulated by transient anchoring of HDL to the plasma membrane through the association of amphipathic helices of apoA-I. Even if HDL binding proteins identified in the plasma membrane (Graham and Oram 1987) have been considered essential for HDL-membrane interaction, some studies indicate that HDL can interact with a protein-free lipid bilayer (Johnson et al. 1988). Tricerri et al. (1998) studying the influence of the recombinant HDL by measuring leakage on 1-palmitoyl-2oleoylphosphatidylcholine (POPC) and POPC/cholesterol vesicles showed the ability of rHDL to interact even with a cholesterol-free lipid bilayer. The results presented here are also not in contradiction with the experiments of Vojčíková and Sabo (2001), which showed the tendency of HDL to increase the resistance and to decrease the capacitance of s-BLM deposited on Pt support.

\section{Acknowledgements}

This work was supported by the Grant Agency of the Slovak Ministry of Education and the Slovak Academy of Sciences 1/3403/06.

\section{References}

BORDI F, CAMETTI C, GLIOZZI A: Impedance measurements of self-assembled lipid bilayer membranes on the tip of an electrode. Bioelectrochemistry 57: 39-46, 2002.

CALABRESI L, GOMARASCHI M, ROSSONI G, FRANCESCHINI G: Synthetic high density lipoproteins for the treatment of myocardial ischemia/reperfusion injury. Pharmacol Ther 111: 836-854, 2006.

CARLSON JW, JONAS A, SLIGAR SG: Imaging and manipulation of high-density lipoproteins. Biophys J 73: 1184$1189,1997$.

COSTELLO RF, PETERSON IR, HEPTINSTALL J, WALTON DJ: Improved gel-protected bilayers. Biosens Bioelectron 14: 265-271, 1999.

DING SJ, CHANG BW, WU CHCH, LAI MF, CHANG HCH: Electrochemical evaluation of avidin-biotin interaction on self-assembled gold electrodes. Electrochim Acta 50: 3660-3666, 2005.

GRAHAM DL, ORAM JF: Identification and characterization of a high density lipoprotein- binding protein in cell membranes by ligand blotting. J Biol Chem 262: 7439-7442, 1987.

JOHNSON WJ, MAHLBERG FH, CHACKO GK, PHILLIPS MC, ROTHBLAT GH: The influence of cellular and lipoprotein cholesterol contents on the flux of cholesterol between fibroblasts and high density lipoprotein. $J$ Biol Chem 263, 14099-14106, 1988.

LU XD, OTTOVA AL, TIEN HT: Biophysical aspects of agar-gel supported bilayer lipid membranes: A new method for forming and studying planar bilayer lipid membranes. Bioelectroch Bioener 39: 285-289, 1996.

MATSUNO Y, OSONO CH, HIRANO A, SUGAWARA M: Single-channel recordings of gramicidin at agarosesupported bilayer lipid membranes formed by the tip-dip and paintings methods. Anal Sci 20: 1217-1221, 2004.

MacDONALD RJ. Impedance Spectroscopy. Emphasizing Solid Materials and Systems. John Wiley \& Sons, New York, 1987, pp. 346.

MUELLER P, RUDIN DO: Action potentials induced in a bimolecular lipid membrane. Nature 217: 713-719, 1968.

NOFER JR, BEATE KEHREL B, FOBKER M, LEVKAU B, ASSMANN G, ECKARDSTEIN A: HDL and arteriosclerosis: beyond reverse cholesterol transport. Atherosclerosis 161: 1-16, 2002. 
NOVOTNY I, REHACEK V, TVAROZEK, NIKOLELIS DP, ANDREOU VG, SIONTOROU CG, ZIEGLER W: Stabilized bilayer lipid membrane on agar-thin film electrode system support. Mater Sci Eng C5: 55-58, 1997.

SABO J, OTTOVA A, LAPUTKOVA G, LEGIN M, VOJCIKOVA L, TIEN HT: A combined AC-DC method for investigation supported lipid membranes. Thin Solid Films 306: 112-118, 1997.

TIEN HT, OTTOVA A: From self-assembled bilayer lipid membranes (BLMs) to supported BLMs on metal and gel substrates to practical applications. Colloid Surface A 149: 217-233, 1999.

TRICERRI A, CORSICO B, TOLEDO JD, GARDA HA, BRENNER RR: Conformation of apolipoprotein AI in reconstituted lipoprotein particles and particle-membrane interaction: Effect of cholesterol. Biochim Biophys Acta 1391: 67-78, 1998.

VOJČÍKOVÁ L, SABO J: High density lipoprotein - model membrane interaction study. Transactions of the Universities of Košice 4: 7-10, 2001.

WANG J, LIN Q, ZHOU R, XU B: Humidity sensor based on composite material of nano/baTiO ${ }_{3}$ band polymer RMX. Sensor Actuator B 81: 248-253, 2002.

WIEGAND G. Fundamental Principles of the Electric Properties of Supported Lipid Membranes Investigated by Advanced Methods of Impedance Spectroscopy. Shaker Verlag GmbH, Germany, 2000, pp. 165.

WHITE SH. The physical nature of planar bilayer membranes. In: Ion Channel Reconstitution. C MiLLER (ed.), Plenum Press, New York, 1985, pp. 3-35.

\section{Corresponding author}

Michal Legiň, Department of Medical Biophysics, Faculty of Medicine, P.J. Šafárik University, Tr. SNP 1, 04066

Košice, Slovakia. E-mail: legin@lf.upjs.sk 